\title{
A Hundred Thousand Lousy Cats (exploring drawing, Al and creativity)
}

\author{
Barbara Rauch \\ OCAD University \\ OCAD University, Toronto, Canada \\ brauch@faculty.ocadu.ca
}

\author{
Michelle Gay \\ Independent Artist/Researcher \\ Toronto, Canada \\ michelle@steamworks.net
}

\begin{abstract}
This paper introduces a practice-led project that uses the Google Quick, Draw! project and dataset to explore the potential differences of algorithmic machine or digitally constructed drawings, and fictional associative hand-drawings. The authors use both digital 20 -second sketching (the rule set for the Quick, Draw! Project) and more elaborate drawings and collages to then analyse and speculate about the results of these types of visualisations. At this phase of research it seems obvious to label and move the machine drawing to the reductive, the handdrawn to the more complex and associative realm but we seek to unpack this binary. Artificial intelligence and machine-learning are producing a wealth of creative projects, we select a couple of case studies to speak to particular visual artefacts that derive from algorithmic processing. For instance, the (IBM Al) Watson-composed film trailer for Morgan is considered as a creative artefact and looked at for its apparent allure and effect on a creative process. Through this inquiry we contemplate surprises and mistakes that come naturally when producing hand-made works, exploring then, what it means to draw and to work within classification systems in an algorithm-leaning world.
\end{abstract}

Creative rule sets. Machine learning. Associative drawing. Imagination versus algorithm. Surprise. Mistakes. Classification systems. Google Quick, Draw!. Watson movie trailer. Feminist data visualisation.

\section{INTRODUCTION}

Using a practice-led approach we explore aspects of creativity, specifically considering studio activities of drawing and collage-making as a response to Google's global drawing challenge described in their Quick Draw! Al system. While this practice-led research project feels related to modernist work like Sol LeWitt's or other conceptual artists using rule sets and instructions to create artworks, we are in fact aiming to place imagination back into digital platforms and explore the socio-political choices that are made for us within technological systems. Our project initiated with a questioning and hacking of the list of words that Google researchers made public to train their Al draw-bot and we argue, tacitly establishes merit. This database of training words is a set of general, non-specific, non-inclusive, averaged-out nouns. One of our research questions asks whether a new taxonomy is tacitly being formed by this process? And what might the implications be? We are left with a seemingly arbitrary choice of words that seem to indicate some information about the researchers and their social and cultural backgrounds. Yet, Google, clearly signals an intention to be inclusive by publishing the dataset on the open access platform Github.

We begin by comparing the Google Quick, Draw! set of drawings to another library of symbols - The Book of Symbols: Reflections on Archetypal Images. This archive for research in archetypal symbolism has compiled a unique list of images, sorted into five sections, creation and cosmos, plant world, animal world, human world and spirit world. The complexity of this undertaking, and its choice of depiction, resulted in an expansive, yet distinct and subjective accumulation of images. The editors state '[i]t is an evocation of the image as a threshold leading to new dimensions of meaning. Symbolic images are more than data; they are vital seeds, living carriers of possibility.' And, artist, Paul Klee said it succinctly as well "[a]rt doesn't reproduce the visible. It renders visible" (Ronnberg 2010 , p.6). We hope that our research/drawing project will similarly add complexity back into the reductive training set of the Google Quick, Draw! Project, a project designed to teach a computer to draw, to recognise drawn symbols and finally to create a global archive of new vector-based simple 
line drawings. Though, we suggest, how to draw is at the same time simple and complex. Questioning the list of Google words, we noted that they were mainly nouns. Our first approach to this list was to attempt to render the nouns into active verbs, to depict action rather than stillness. Studying these terms further revealed questions in the Al drawing system, their point being to reduce ambiguity in understanding what a drawn form means (for instance car, camouflage, cup). As creative practitioners however, we aim to introduce gaps back into the readings/understanding of the images. This is where humans may find pleasure through open-ended interpretation of images. Whereas when we train Google's Al algorithm through the computer interface this space of poetry becomes somewhat calcified. When we read the words as single entities, we find little poetic resonance. Our methodology then involves a creative re-classification of the terms. We curate Google's terms into simple groupings to then begin our drawing and collage-making process in the studio. We propose that this re-classification allows and welcomes messy resonance with, and between, the original terms and is part of our attempt to unpack the various elements of this example of an Al system.

\section{PROJECT IMPETUS AND BEGINNINGS}

Intrigued by the software platform Google Quick, Draw! BR and MG embarked upon a collaborative research project that took as its starting point our curiosity around training a drawing Al system for some kind of creative use.

As visual artists/makers and as researchers interested in the impact of technologies on creativity and studio work, we tease out aspects of this deceptively simple project through acts of drawing and collaging, and thinking critically about some of the implications of human-machine collaborations in creative projects.

BR and MG are visual artists who have used technology and drawing in past works, and we found there were many rich facets revealed when we began to scratch at the surface of this project. Google Quick, Draw! is a drawing project where humans 'play' a 20-second drawing game. The gamer/drawer is introduced to a single word in a random order, such as airplane, alarm clock, ambulance, etc. You begin drawing on-screen monitored by a countdown timer and a 'system voice' which begins to declare what it 'thinks' you are drawing. "I see mule, I see dog, I see frog, oh I know, it's a cat". The algorithm either figures it out within the 20 seconds, or it informs you that it doesn't understand your drawing. You continue playing the drawing game if desired.
Investigating the public-facing descriptions of Quick, Draw! reveals Google's hopes for this project and by extension we use it as a method to contemplate human-machine collaborations in the domain of artwork, studio work and artistic output.

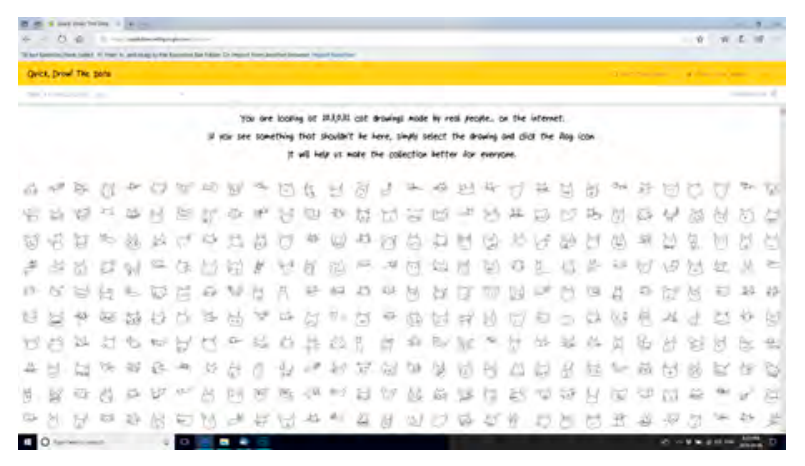

Figure 1: Google Quick, Draw! Interface - Cats

For instance, we conceptualise Google's Quick, Draw! README document as a contemporary vision statement:

The Quick Draw Dataset is a collection of 50 million drawings across [345 categories] contributed by players of the game [Quick, Draw!] (...) The drawings were captured as timestamped vectors, tagged with metadata including what the player was asked to draw and in which country the player was located. You can browse the recognized drawings on [https://quickdraw.withgoogle.com/data).

We're sharing them here for developers, researchers, and artists to explore, study, and learn from.

Then visiting the Quick, Draw! public interface, the question is posed 'What do 50 million drawings look like?'

Over 15 million players have contributed millions of drawings playing Quick, Draw! These doodles are a unique data set that can help developers train new neural networks, help researchers see patterns in how people around the world draw, and help artists create things we haven't begun to think of. That's why we're opensourcing them, for anyone to play with. (Emphasis ours).

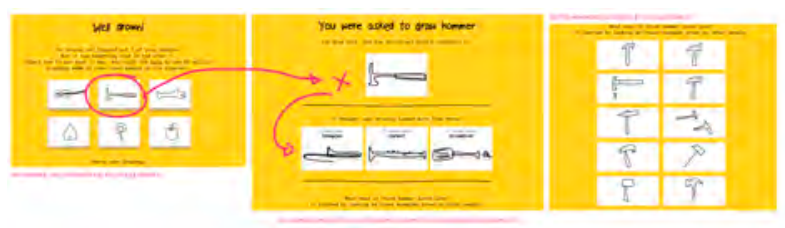

Figure 2: Google Quick, Draw! Interface - Doodles 


\section{A HUNDRED THOUSAND LOUSY CATS \\ Barbara Rauch, Michelle Gay}

\subsection{Methodology: Practice-led Research \& Employing Feminist Data Analysis}

Being creative practitioners we were intrigued by Google's references to creativity and the work of artists. In fact helping artists create things we haven't begun to think of is an interesting proposition, and a challenge that we couldn't resist. We framed our investigation within this system. Firstly we doodled using the online platform, we then reviewed the online set of drawings produced by anonymous users training the $\mathrm{Al}$, then we downloaded the training set of terms to use in our studio exploration. After downloading we reviewed, contemplated and interrogated the list of terms and words.

This lead to some early questions:

- Reviewing the categories, the language used in training the draw-bot system, why these particular words?

- Why not verbs? Why not actions?

- What does it mean to draw and what does it mean to draw reductively in a 20 -second doodle?

- Where do these synthesised drawings (doodles) get used by Google?

- How would artists use these doodles within a studio practice?

To address this expanded set of questions arising from the primary research and our drawing and collaging efforts, we use some feminist principles outlined by Catherine D'Ignazio and Lauren Klein in their online paper Feminist Data Visualization (D'Ignazio \& Klein 2016). They apply feminist theory and methods to data visualisation research to introduce and mingle digital humanities approaches with information design. Their dictum is: data, design and, community use of the data, are all intertwined.

The six principles of feminist review of technologies are:

(i) rethink binaries;

(ii) embrace pluralism;

(iii) examine power \& aspire to empowerment;

(iv) consider context;

(v) legitimise embodiment and affect;

(vi) make labour visible.

We use these principles to further structure our research process and findings. In this paper we also briefly touch on: the politics of language; look at ubiquitous computing (ubicomp); consider machine-human creative collaborations; explore the act of training Al systems; and consider sociopolitical implications of classifications and categorisations (in general).
Table 1: Sample of Drawn.Categories. Google Gay/Rauch 2017

\begin{tabular}{|c|c|c|c|}
\hline \multirow[t]{2}{*}{$\begin{array}{c}\text { Original } \\
\text { Order }\end{array}$} & \multicolumn{3}{|c|}{ 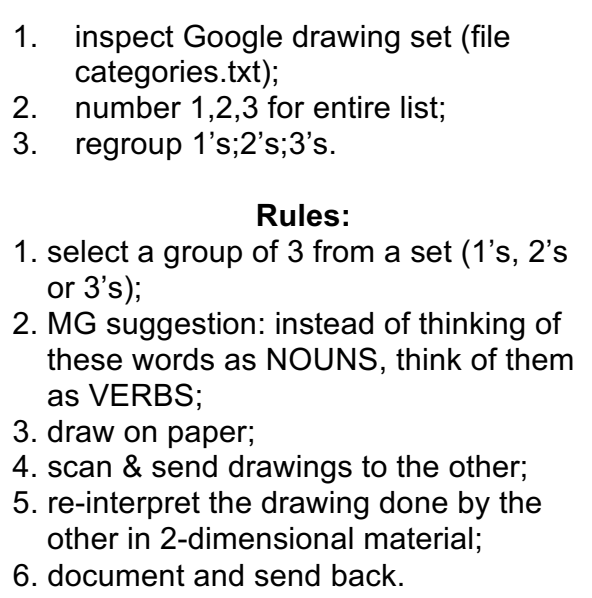 } \\
\hline & 1's & 2's & 3's \\
\hline $\begin{array}{l}\text { aircraft } \\
\text { carrier }\end{array}$ & $\begin{array}{l}\text { aircraft } \\
\text { carrier }\end{array}$ & airplane & alarm clock \\
\hline airplane & ambulance & angel & $\begin{array}{l}\text { animal } \\
\text { migration }\end{array}$ \\
\hline alarm clock & ant & anvil & apple \\
\hline ambulance & arm & asparagus & axe \\
\hline angel & backpack & banana & bandage \\
\hline $\begin{array}{l}\text { animal } \\
\text { migration }\end{array}$ & barn & baseball & baseball bat \\
\hline ant & basket & basketball & bat \\
\hline anvil & bathtub & beach & bear \\
\hline apple & beard & bed & bee \\
\hline arm & belt & bench & bicycle \\
\hline asparagus & binoculars & bird & birthday cake \\
\hline axe & blackberry & blueberry & book \\
\hline backpack & boomerang & bottlecap & bowtie \\
\hline banana & bracelet & brain & bread \\
\hline bandage & bridge & broccoli & bucket \\
\hline \multicolumn{4}{|l|}{ barn } \\
\hline \multicolumn{4}{|l|}{ baseball } \\
\hline Etc. & & & \\
\hline
\end{tabular}

After inspecting the Google drawing word set and keeping in mind that we are working towards "helping artists create things we haven't yet begun to think of" we took a decision to re-interpret the given rules, using our own creative rule-set to see what would emerge. Our methods involved rearranging the ordering of terms and creating new word combinations for our own drawings and collages. Michelle interpreted the new rules slightly different to Barbara who selected one word from 
each category to make one drawing, while $\mathrm{M}$. selected sets of three words from one category. We scanned and documented our drawings and sent them to the other (in Canada or Scotland). The drawings or collages are different and associative, in contrast to our doodle machine drawings where words are depicted in straightforward vector sketches. In our studio works we allow interpretation and association to influence the list of words and the image creations. We intuitively respond to our own gestures and allow ink to take her own route, which subsequently triggers additional gestures on paper.

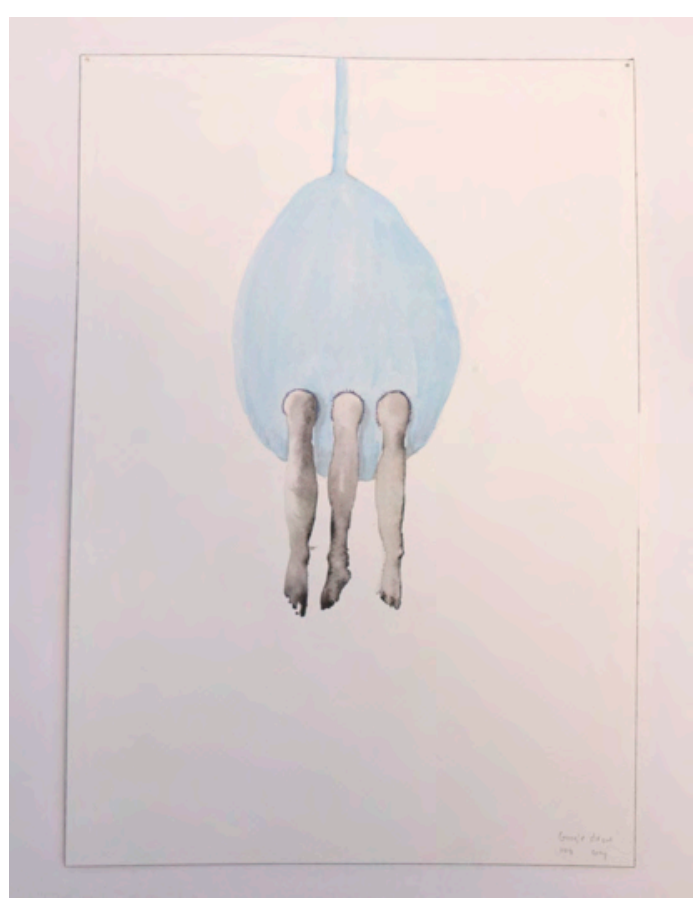

Figure 3: Knee Lantern Leg. 2017. M. Gay

The 345 words on Google's list are nouns that someone decided are words worth drawing for the initial Al training. As part of our investigation we created some different lists that we felt more inclusive and offered opportunities for creative interpretation.

Data visualisation projects, generally speaking, aim to make large data sets accessible usually by cleaning up some of the data. As artists we rather liked the messiness of our data. As M. put it, "we embrace surprise, delight and challenge, definitely three aspects to look for in the world as active and present humans". Google Quick, Draw! at first glance doesn't like messiness; it aims for the perfect drawing, distinct and quickly recognisable. A closer look however reveals that all the messiness remains in their database to calculate the average drawing.

\subsubsection{Computational Creativity \& Ubiquitous Computing}

A few more questions arise around our use of technologies, platforms and systems. Mark Weiser (1991) coined the term 'ubiquitous computing' during the early 1990s, he reminded us then how technologies are woven into everyday life, and when they become indistinguishable from life we ought to be somewhat wary. Since these early days, much has changed and we could argue we have entered the next phase where Al software algorithms are starting to make decisions for us. We are giving control to Al, for instance when a fitness watch calculates how many more steps are needed to take to be a healthy person. We are also potentially handing over creative control with systems such as Google Quick, Draw!.

While the premise of the drawing game seems like a collaboration between human and machine, we are left with the question of who is training whom. The Al (male) voice that tells Michelle what she supposedly draws or, Michelle's 20-second drawings of what we cheekily call 'lousy cats' that are integrated into a database?

We are reminded of AutoCAD software aimed for architects to sketch more quickly. Yet, a story by architect Renzo Piano describes why drawing by hand is important to his creative process "[y]ou start by sketching, then you do a drawing, then you make a model, and then you go to reality - you go to the site - and then you go back to drawing. You build up a kind of circularity between drawing and making and then back again" (Sennett 2008, p. 40).

It is the revisiting, the redoing, the dwelling in the space of imagination, that we need to practice, while AutoCad uses an efficient and auto-corrective system that doesn't ask you back in, in the same way. Sennett recounts: 'physicist Victor Weisskopf once said to his MIT students who worked exclusively with computerized experiments, "When you show me that result, the computer understands the answer, but I don't think you understand the answer"' (Sennett 2008, pp. 40 -41).

We argue that being auto-corrected by any of these machine systems will not train you to do a better drawing. Our concern is that it might even make you passive, less observant, and less engaged with the forms you are attempting to create, draw or compose.

Again it is the surprise and space to contemplate, that we look for in creative practices. If we are asked to collaborate with intelligent systems, should there also be a discussion with users about these tools they are being asked to train and use? We are thinking critically of Quick, Draw! as an 
early Al system that involves people in a 'gamified' way. We propose that, as a culture, we should be having discussions about these tools, machines and processes as they develop. Without transparencies around development of new tools, which in the future could be used globally and ubiquitously, we run the risk of tacitly accepting new tools integrated into our lives without any chance to reflect, have input, discuss as a culture, and as D'lgnazio (2017) suggests, 'make dissent possible'.

This principle D'Ignazio (2017) presents is attractive to us as artists, as creative individuals, as critical users of technologies in life and in our studio work. We might interpret 'dissent' to also mean being a 'reflective practitioner' (Schön 1983). But the question is where to insert this dissent?

\section{Catherine D'Ignazio asks}

How can we devise ways to talk back to the data? To question the facts? To present alternative views and realities? To contest and undermine even the basic tenets of the data's existence and collection? (...) It might be as simple as including different people, with different perspectives, in the production of the visualization. (D'Ignazio 2017).

We would add, include people in the production of the tools, platforms, technologies, and classification systems.

There are many scholars thinking about these issues of human-machine relations, coding, and the interconnected topics of categorisations and classifications. For example Lucy Suchman (1993) re-frames Langdon Winner's (1980) question about technology and ethics - Do Artifacts have Politics? to Do Categorizations have Politics? And other scholars like Lucas Introna and Helen Nissembaum (Introna \& Nissenbaum 2000) consider how we might help shape ubiquitous tools such as software platforms. As D'Ignazio, Klein and other scholars propose, we want to be involved in helping conceptualise, code, and categorise our current and future tools. So, do categories have politics? Thinking of D'Ignazio and Klein's feminist principles of data visualisation we would answer "yes" to this question and to Langdon Winner's original query 'do artifacts have politics'. Unpacking this, we dive a little deeper into intelligent systems, we see that training the system is an important step along the path for Al understanding.

In our example of Quick, Draw!, the initial training is multi-fold and involves: a set of words which are referred to as 'categories'; the code which captures a 20-second vector drawing on a screen; and the storing in a database of comparable 20-second vector drawings. We asked earlier, where did these 'categories' come from? Why these words and not other words?

This seems like a good place to circle back to our discussion as artists and creative practitioners. We are both interested in these types of collaborations between humans and machines, and have explored this topic within our studio work.

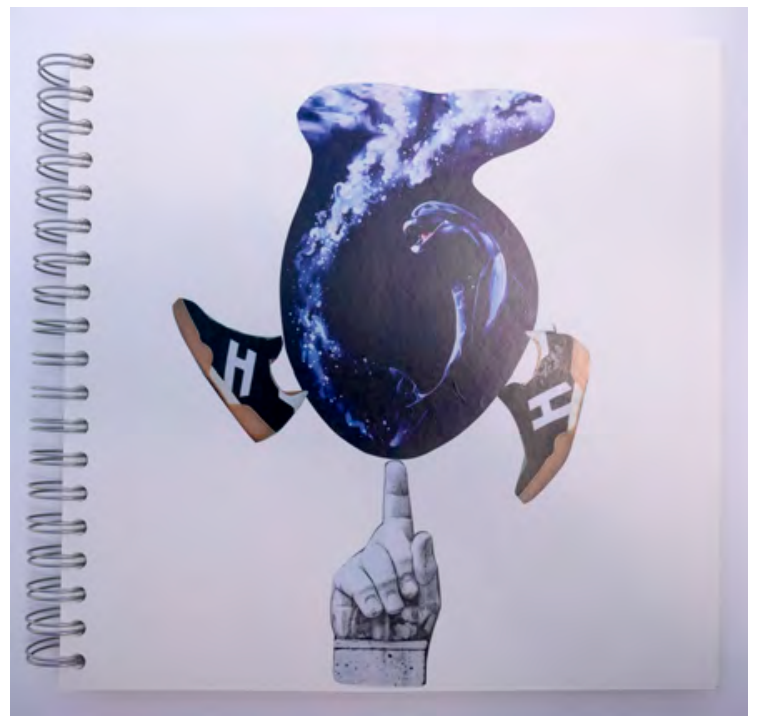

Figure 4: Shoe Hand Dolphin. 2018. B. Rauch

\subsubsection{Creative artefacts produced by computational means}

Looking for examples of creative endeavours with $\mathrm{Al}$ systems we compare another use of an $\mathrm{Al}$ system to produce creative artefacts. Namely IBM's Watson intelligent system that was used to produce the movie trailer for a sci-fi film called Morgan in 2016. In this case the creators of the system use the term 'augmented intelligence'.

Continuing then with our investigation around creativity and machines, we offer here a short discussion about 'the first-ever machine human collaboration at creating a real movie trailer' (Smith et al. 2017). This augmented intelligent system was developed by researchers at the IBM Watson Research centre in conjunction with the visiting researchers from the National Taiwan University and the support of $20^{\text {th }}$ Century Fox movie studios.

Our interest here is to think about machines and creativity, so this tool/platform also caught our attention. We ask these questions: a) What does computational creativity suggest? b) What are the potential cultural artefacts? c) How might it change an artist's studio practice? We look at the components of this project to help us consider these questions and implications. Unlike the Quick, Draw! though, there is no public-facing component for us to play with. 
This 'intelligent system' was designed to review segments or snippets of the film Morgan to then determine instructions for creating the best trailer for this sci-fi film without giving away the ending of the film. Other requirements included thinking of the potential emotional impact of a scene, and to deliver an aesthetic experience that would be similar to the actual film. One important detail, the intelligent system selected 10 clips that, given its working parameters, would make good candidates for a film trailer, it did not produce the final artefact. A human film editor, presumably with much experience in editing, completed this 2:24-minute short film (https://youtu.be/Dc5BE8iwmHw). The system wasn't starting from scratch to create a storyline, a narrative, a sound track, or final edited film at this point in time. Exploring the mechanics behind the intelligent system, we understand that much 'training' went into the system before it was introduced to the potential segments of Morgan.

As the researchers tell us:

\begin{abstract}
the system has been trained on horror trailers from the top 100 horror movies by segmenting out each scene from the trailers and performing audio-visual analysis including visual sentiment and scene analysis of visual key-frames, audio analysis of the ambient sounds such as character's tone of voice and musical score, to understand the sentiments associated with each of those scenes, and the composition (including shot location, image framing and lighting). (Smith et al. 2017, p.2)
\end{abstract}

In short, the system also needs to be trained to do its creative job just as Google's Quick Draw! system. The researchers interestingly employ many components such as large-scale audio visual and textual databases. (Some examples are, MediaEval Benchmarking initiatives that identify violent scenes; OpenSMILE project for audio-based sentiment; MovieQA dataset with 400 movies for plots, subtitles; MovieBook Dataset connecting books to films; MPII Movie Description dataset for visually impaired people; OpenEar dataset which provides characteristics culled from audio tracks). These and about half a dozen other systems were employed.

At this point in our research we are not reviewing the datasets, we will do this in the next phase of our project. Here we simply note that these types of collections (datasets) have been employed in the making of this trailer.

Being mindful of the questions that scholars Suchman and Winner propose: do categories have politics and do artefacts have politics? Our interest is to think about the politics of these types of datasets. Here, the intelligent system, algorithmically combines dataset content to employ details such as emotional classes (anger, disgust, fear, happiness) or emotional states (boredom, cheerful, nervous, surprise) (Smith et al. 2017). And for visuals the development and research team employed classification tools like Robert Plutchik's Wheel of Emotion from 1980 and a dataset called SentiBank developed at Columbia University's Digital Video and Multimedia Lab.

A summary note published on the Columbia University's SentiBank - Visual Sentiment Ontology site states:

The analysis of emotion, affect and sentiment from visual content has become an exciting area in the multimedia community allowing to build new applications for brand monitoring, advertising, and opinion mining. (...) This database contains a Visual Sentiment Ontology (VSO) consisting of 3244 adjective noun pairs (ANP), SentiBank a set of 1200 trained visual concept detectors providing a mid-level representation of sentiment, associated training images acquired from Flickr, and a benchmark containing 603 photo tweets covering a diverse set of 21 topics. (Columbia University n.d.).

Researchers Smith et al. (2017) delve into literary theory, describing their discovery of the potential use of 'tropes' for further contextual understanding. Along with all the other datasets mentioned, they employ a trope dataset from a trope wiki (TV Tropes, n.d.). Tropes, as they write in their paper are 'figures of speech'. We understand tropes as storytelling devices used in the crafting, creation and contextualization of narratives. 'A trope is a storytelling device or convention, a shortcut for describing situations the storyteller can reasonably assume the audience will recognize. (...) It's pretty much impossible to create a story without tropes' (Smith et al. 2017).

The researchers here employ the human concept 'trope' pulled from a trope-wiki, used alongside a suite of other datasets within this new intelligent system to produce a creative artefact. It appears that human fingerprints are all over these intelligent systems. Continuing with our feminist questioning of data, data collection, data organisation and data provenance we circle back to D'Ignazio and Klein's (2016) inquiries regarding the material economy behind the data. Highlighting some questions,

- Who are the funders?

- Who collected the data?

- Whose labour happened behind the scenes and under what conditions?

D'Ignazio proposes a methodology: 'What if we visually problematized the provenance of the data, the interests behind the data, and the stakeholders in the data?' (D'Ignazio 2017). We too are interested in knowing more about the collecting 
processes, how categories are derived and who took these decisions. D'Ignazio further suggests that collecting and displaying this type of metadata may be an interesting tactic. The goal of revealing such visualisations would be to show 'what the data says' and to also 'show how the data connects to real bodies, systems and structures of power in the wider world' (D'Ignazio 2017).

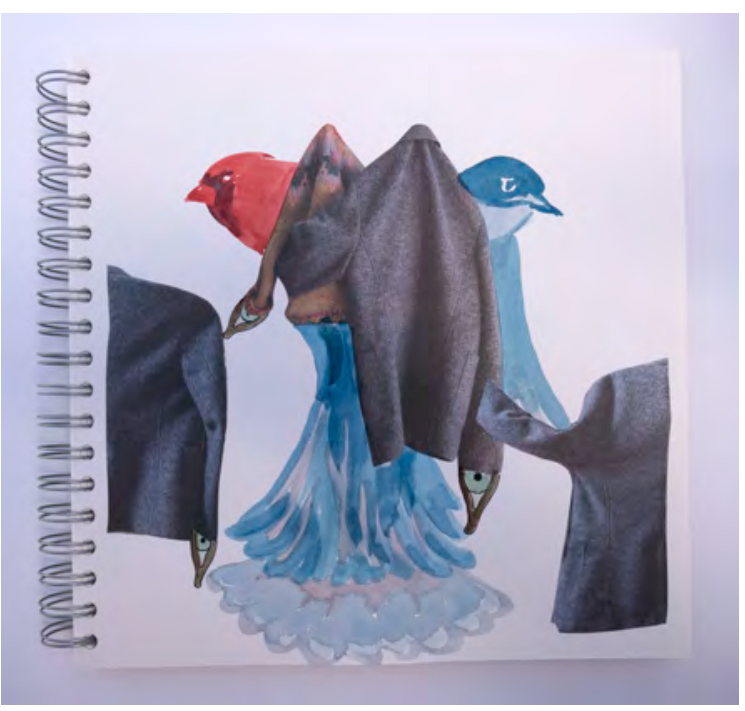

Figure 5: Bird Jacket Eye. 2018. B. Rauch

This connects back to our initial discussion about Quick, Draw! and the extended library of Google Al experiments. As users of the system and creative practitioners, it is not clear what we are really training the draw-bot to do. Is it to understand and to categorise 'lousy cats' - those 20 second doodles? Or, is there some larger purpose not identified by Google at work here? We continue to wonder about the creative use by artists of Quick, Draw! or the computational creativity of Watson as a new type of storyteller.

Summarizing, we have been questioning the set of words to train Quick, Draw! and review the sociopolitical resonances of training algorithms to produce cultural artefacts. Through this practice-led research we reveal and pose new questions around the entanglement of humans and machines in the making of creative artefacts. There is no finding of a tidy conclusion, so we pose more questions: Why does Google want to train an Al system to draw these words? Why these words and not more inclusive words? What is the global database of 20-second drawings going to be used for? Will these be used as a ubiquitous representation of 'car, cup, cat, arm, saw'? What gets lost in the doodle process? What are the dangers of this reductive list and database of drawings?
Comparing this process to citizen science gamified systems and platforms we see a difference in that people embark on tasks with an understanding of the project impetus and the context for their research findings. A current example is the transparency seen in the experiment Gravitational Lens image classification project (Space Warps HSC n.d.). Here the distributed network of participants will all be listed as researchers on the project if and/or when discoveries are made. This demonstrates one of our feminist principles, namely 'make labour visible'.

We loop back to the concepts of training systems for our creative uses. In both cases of Quick, Draw! and the Morgan trailer, we see that these systems still need humans as participants, as drivers, as trainers, as classifiers, as category-makers. Another interesting component employed to help categorise sentiment and language for the Morgan trailer is the extensive work around adjective-noun pair sets (anps) mentioned by SentiBank (Columbia University n.d.). This brings us to concepts like 'happy dogs' and 'grumpy cats'. Similar to our reclassification and word grouping methods, an adjective-noun pair set also complicates the original Quick, Draw! word set. We want to expand and enhance the drawing database of cats, adding for instance, top view, belly view, paw view, grumpy cat, hungry cat, cat that just bit Barbara, cat that will grudgingly accept to sit on your lap and allow you to scratch their ears.

This reminds us of a story that Richard Sennett recounts in The Craftsman around the concepts of language, open narratives and interpretation. Sennett (2008) further speaks to the richness and potential connection of language and material representations. He recounts the story of how Pope Sixtus V re-built the Piazza del Popolo in Rome at the end of the 16th century 'by describing in conversation all the buildings and public space he envisioned', he used 'verbal instructions that left much room for the masons, glaziers and engineers to work freely and adaptively on the ground' (Sennett 2008, p. 41). Meaning, the crafts and creative workers interpreted and materialised his narrative as they understood it from his description.

\subsubsection{Preliminary Findings \& Further Research. Giving over to algorithms}

We approached Quick, Draw! as a potential new tool to 'imagine new things not yet thought of' (Google ReadMe, n.d.). We try and play the game, yet are compelled to add complexity and messiness to our sketches or doodles. This does not go over very well with the draw-bot. As a creative practitioner using this system, we rhetorically ask if we learned new things about drawing? Thinking here of Renzo Piano's urging to 
use drawing to understand the thing you are thinking about (Sennett 2008).

We appreciate D'Ignazio and Klein's (2016) input to this inquiry, calling their six principles a preliminary list. We take up the challenge to expand, use and test these principles when thinking of humanmachine collaborations.

Our manipulation of the Google word list, the new constellations we drew and collaged are in line with the criticality our feminist principles call for. We didn't trust the rules we were given, we searched for lost or 'failed' drawings in the Google database, and allowed drifting, chance, and new associations to offer alternative narratives. A critical game cannot anticipate the user's intention, and as artists we do ask questions; the condition of some of the most compelling art plays with the potential of failure, revels in the unclassified and the unforeseen and so challenges and includes the viewer's intelligence in layered readings.

So far we have used the word 'drawing' and 'doodling' synonymously yet we understand a doodle more as an absent-minded action, while sketching, drafting, illustrating etc. address professional drawing practices that are skills of experts. We would like to mention a couple of artists addressing contemporary drawing practice, for instance Grayson Perry thinks that '[u]ntil we can insert a USB into our ear and download our thoughts, drawing remains the best way of getting visual information on to the page (...)' (Perry n.d.). Or Dawn Brennan from The Chicago School of Media Theory says it ever so eloquently "[a]lthough I treat "drawing" here in its artistic sense - as the act or product of the act of making marks, designs or tracings on a surface - I am glad to be reminded that the word "draw," as in the sense of a horse drawing a cart, means to drag, pull, or bear. The word's etymology cannot be broken down any further' (Brennan 2002).

To conclude we share a short anecdote from our foundation years in the academy, when $B$ was asked to do still life drawings of a cauliflower, her professor stopped her right away and said you should really eat a bit of that raw cauliflower before you start your drawing, you will understand better its consistency, how crunchy and hard it is and how it tastes. Many other tricks were handed down that helped undo assumptions that we carry with us. To draw a cauliflower or a cat with a computer mouse is still another discussion. In the 20 seconds we have, we are asked to reduce the cauliflower to its most distinct features, the cat to what makes it look most 'catlike'. Lingering with our new word combinations, allowing our lines and forms to drift, opens a space to create and interpret image sets. We assume that our methodological use of
Google's drawing challenge was not the machinic interpretation expected, although it did 'help us', as artists, 'create things we hadn't yet begun to think of'. Using our practice-led research method to take up Google's challenge has spurred more questions for us as creative practitioners. We continue to wonder what makes an interesting drawing and ask how will neural networks recognize the complexity that makes a drawn form successful.

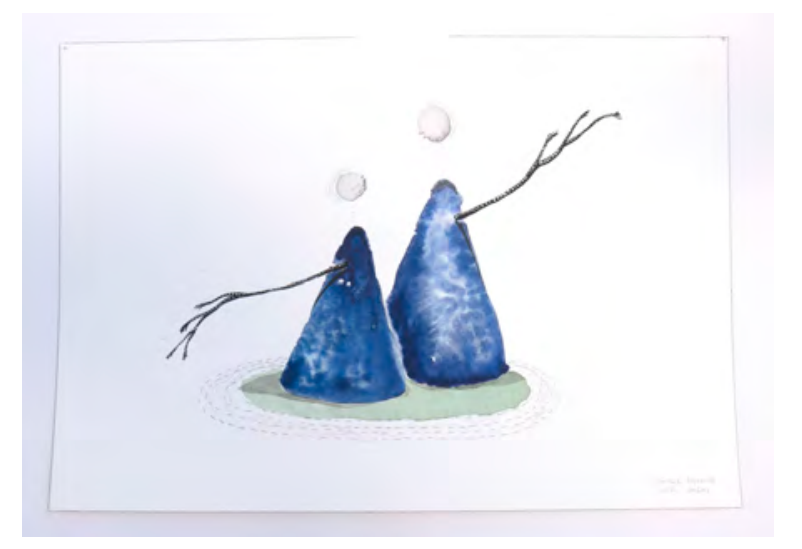

Figure 6: Moon Mountain Mouth. 2017. M. Gay

\section{REFERENCES}

Brennan, D. (2002) drawing. The Chicago School of Media Theory. University of Chicago. Available from:

https://lucian.uchicago.edu/blogs/mediatheory/keyw ords/drawing/ [June 10, 2018].

Columbia University, Digital Video and Multimedia Lab. (n.d.) SentiBank - Visual Sentiment Ontology. Available from:

www.ee.columbia.edu/ln/dvmm/vso/download/senti bank.html [June 10, 2018].

D'Ignazio, C. (2017) What would feminist data visualization look like? Medium. Available from: https://medium.com/@kanarinka/what-wouldfeminist-data-visualization-look-like-aa3f8fc7f96c [June 10, 2018].

D'Ignazio, C. \& Klein LF. (2016) Feminist Data Visualization. In IEEE VIS Conference, Baltimore, Maryland, October 2016. IEEE, New York.

Google (n.d.). AutoDraw. Google. Available from: https://www.autodraw.com [June 10, 2018].

Google (n.d.) Quick, Draw! Google. Available from: https://quickdraw.withgoogle.com [June 10, 2018].

Google (n.d.) README.md. Google. Available from:

https://github.com/googlecreativelab/quickdrawdataset [June 10, 2018]. 
Introna, L.D. \& Nissenbaum, H. (2000) Shaping the Web: Why the Politics of Search Engines Matters. The information Society. 16:3, pp. 169-185.

Morgan Official Trailer \#1. Available from: https://youtu.be/Dc5BE8iwmHw [June 10, 2018].

Perry, G. (n.d.) What is Drawing? Victoria \& Albert Museum. Available from:

http://www.vam.ac.uk/content/articles/w/what-isdrawing/ [June 10, 2018].

Ronnberg, A. (2010) The Book of Symbols, Reflections on Archetypal Images. Taschen, Köln.

Schön, D. (1983) The Reflective Practitioner: How Professionals Think in Action. Basic Books, New York.

Smith, J.R., Joshi, D., Huet, B., Hsu, W., \& Cota, J., (2017) Harnessing Al for Augmented Creativity: Application to Movie Trailer Creation. MM '17 ACM. Mountain View, CA. October 23 - 27, 2017. DOI: 10.1145/3123266.3127906 [June 10, 2018].
Sennett, R. (2008) The Craftsman. Yale University Press, New Haven \& London.

Space Warps - HSC. (n.d.) Searching for strong gravitational lenses in the Hyper Suprime-Cam (HSC) survey. Available from:

https://www.zooniverse.org/projects/aprajita/spacewarps-hsc [June 10, 2018].

Suchman, L. (1993) Do Categories Have Politics? Computer Supported Cooperative Work (CSCW) 2: $177-190$.

TV Tropes. (n.d) Tropes. Available from: http://tvtropes.org/pmwiki/pmwiki.php/Main/Tropes [June 10, 2018].

Weiser, M. (1991) The Computer for the $21^{\text {st }}$ Century. Mobile Computing and Communications Review, Vol 3, Number 3.

Winner, L. (1980) Do Artifacts have Politics? Daedalus, Modern Technology: Problem or Opportunity? 109, No. 1, pp. 121-136. 